\title{
A High-Resolution Silicon-on-Glass $Z$ Axis Gyroscope Operating at Atmospheric Pressure
}

\author{
Haitao Ding, Xuesong Liu, Longtao Lin, Xiaozhu Chi, Jian Cui, Michael Kraft, Zhenchuan Yang, and \\ Guizhen Yan
}

\begin{abstract}
This paper describes a high-resolution silicon-onglass $z$ axis gyroscope operating at atmospheric pressure. The mechanical structure is designed in such a way that it exhibits low cross coupling between drive and sense mode of less than $0.5 \%$ simulated using finite-element method and $1.35 \%$ verified by experimental measurements. Due to a symmetrically designed structure, the specified bandwidth can be maintained despite of fabrication imperfections. The fabrication process flow is based on a combination of silicon on glass bonding and deep reactive ion etching which results in a large proof mass and capacitances. A closed loop self-oscillation drive interface is used to resonate the gyroscope in the drive mode, which reaches steady-state after $150 \mathrm{~ms}$. Using area-varying capacitors, large quality factors of 217 and 97 for drive and sense mode, respectively, were achieved operating at atmospheric pressure. A low drive voltage, with a $1 \mathrm{~V}_{\text {peak-peak }}$ AC drive amplitude and $10 \mathrm{~V}$ DC bias was used to excite the drive mode. The measured scale factor was $10.7 \mathrm{mV} /{ }^{\circ} / \mathrm{s}$ in a range of $\pm 300^{\circ} / \mathrm{s}$ with a $\boldsymbol{R}^{2}$-nonlinearity of $0.12 \%$. The noise equivalent angular rate is $0.0015^{\circ} / \mathrm{s} / \mathrm{Hz}^{1 / 2}\left(=5.4^{\circ} / \mathrm{h} / \mathrm{Hz}^{1 / 2}\right)$ in a $50 \mathrm{~Hz}$ bandwidth. The measured SNR was $34 \mathrm{~dB}$ at an angular rate input signal with an amplitude of $12.5^{\circ} / \mathrm{s}$ and a frequency of $10 \mathrm{~Hz}$. Without any active temperature control, zero bias stability of $1 \% / \mathrm{s}$ was achieved for long-term measurements over six hours and $0.3^{\circ} / \mathrm{s}$ for short-term measurements over 120 seconds $(1-\sigma)$.
\end{abstract}

Index Terms-Atmospheric pressure, closed-loop, double decoupled, gyroscope.

\section{INTRODUCTION}

$\mathbf{M}$ ICROELECTROMECHANICAL SYSTEMS (MEMS) gyroscopes have received worldwide attention due to their numerous advantages such as small size, reduced power consumption, and low cost. Since the latter is crucial for mass market applications, in recent years, considerable efforts have been put into the development of MEMS gyroscopes being able to operate at atmospheric pressure; this further decreases

Manuscript received June 29, 2009; revised October 18, 2009; accepted February 05,2010 . Current version published April 02, 2010. This is an expanded paper from the IEEE SENSORS 2008 Conference. The associate editor coordinating the review of this paper and approving it for publication was Dr. Venkat Bhethanabotla.

H. T. Ding, X. S. Liu, L. T. Lin, X. Z. Chi, J. Cui, Z. C. Yang, and G. Z. Yan are with the Institute of Microelectronics, Peking University, Beijing 100871, China (e-mail: dinght@ime.pku.edu.cn; liuxuesong@ime.pku.edu.cn; linlongtao@ime.pku.edu.cn; xzchi@ime.pku.edu.cn; cuijian@ime.pku.edu.cn; z.yang@ime.pku.edu.cn; gzyan@ @ku.edu.cn).

M. Kraft is with the School of Electronics and Computer Science, University of Southampton, Southampton, SO17 1BJ, U.K. (e-mail: mk1@ecs.soton.ac. uk).

Color versions of one or more of the figures in this paper are available online at http://ieeexplore.iee.org.

Digital Object Identifier 10.1109/JSEN.2010.2043669 the unit cost since vacuum packaging is often the most expensive step in the fabrication process. In the following, the performance characteristics of MEMS gyroscopes operating at atmospheric pressure are briefly summarized. Kim et al. reported a combined surface and bulk-micromachined electromagnetic gyroscope with a resolution of $0.15^{\circ} / \mathrm{s}$ and noise equivalent rate of $0.05^{\circ} / \mathrm{s}$ in a $10 \mathrm{~Hz}$ bandwidth [1], and a bulk-micromachined single crystal silicon gyroscope with a noise equivalent level of $0.05^{\circ} / \mathrm{s}$ in a dynamic range of $\pm 100^{\circ} / \mathrm{s}$ [2] working at atmospheric pressure. Xie et al. described a CMOS-MEMS gyroscope achieving a noise floor of $0.02^{\circ} / \mathrm{s} / \mathrm{Hz}^{1 / 2}[3]$. Xiong et al. reported a "slots gyroscope" [4] with $Q$-factors of 100 and a tuning fork gyroscope [5] with $Q$-factors of nearly 1000 , but no further results about resolution and bias stability were reported. Acar et al. proposed a robust micromachined gyroscope with a 2-DOF sense-mode oscillator and a measured noise floor of $0.64^{\circ} / \mathrm{s} / \mathrm{Hz}^{1 / 2}$ [6]. Alper et al. reported a high-performance SOI (silicon on insulator)-MEMS gyroscope with a measured noise equivalent rate of $90^{\circ} / \mathrm{h} / \mathrm{Hz}^{1 / 2}$ and bias stability of $1.5^{\circ} / \mathrm{s}$ for $100 \mathrm{~s}$ [7], and in another publication a gyroscope with a resolution of $0.030^{\circ} / \mathrm{s}$ in $50 \mathrm{~Hz}$ bandwidth [8]; the latter device has a similar mechanical design to the device described in this work, but with a structural thickness of only $12-15 \mathrm{um}$. We previously described a bulk-machined gyroscope with an open-loop drive scheme, achieving a noise equivalent rate of $0.0084^{\circ} / \mathrm{s} / \mathrm{Hz}^{1 / 2}$ [9].

Although substantial progress in the development of non-vacuum packaged gyroscopes has been achieved, the sensor characteristics only meet the requirements of low performance applications. In this paper, we propose a doubledecoupled silicon-on-glass gyroscope, with a considerably improved resolution compared to previously described gyroscopes operating in air, and therefore aiming to satisfy some applications with medium-precision requirements, such as robotics, miniature inertial measurement unit (MIMU), and short time GPS bridging. The approach uses two groups of one-degree-of-freedom (1DOF) cantilevers to minimize the mechanical cross coupling between drive and sense mode, and employs area-varying capacitors to achieve large quality factors at atmospheric pressure. A symmetric structure is designed to maintain the specified bandwidth despite of fabrication imperfection. Parts of this work have been published in [10].

\section{Structural Mechanical Design}

Fig. 1 shows the schematic diagram of the mechanical design of our gyroscope. The mechanical structure is symmetric, 


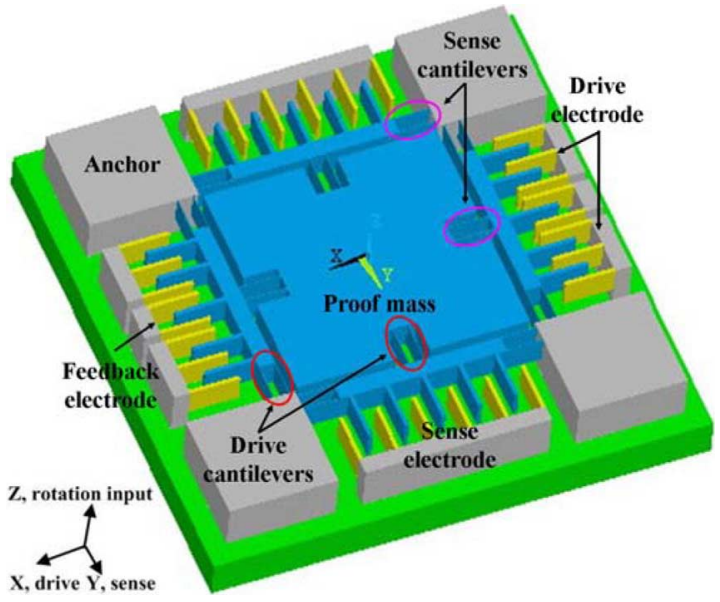

Fig. 1. Schematic diagram of the proposed gyroscope.

which results in desirable matching of the resonant frequencies of drive and sense mode. It also substantially reduces susceptibility to environmental parameters, e.g., temperature drift [8], and to fabrication tolerances. Ideal frequency matching results in maximum sensitivity; alternatively one can implement a design which deliberately shifts the drive mode from the sense mode resonant frequency to obtain good environmental parameter robustness, stability, and high bandwidth. Either can be easily achieved by simply changing the width of the suspension cantilevers; therefore the proposed design, due to its symmetry, is suitable to be adopted for a variety of applications.

The two-degree-of-freedom (2DOF) proof mass is suspended by two groups of cantilevers and compliant along the two in-plane axes; one each for drive and sense mode. Each group consists of 12 cantilevers which are compliant along one axis only. The excited drive motion along the $x$ axis does not result in a motion along the sense axis, and likewise Coriolis induced motion along the $y$ axis does not result in any motion along the $x$ axis. In this way, a double decoupling scheme is realized between the two operating modes and hence achieves good mechanical cross coupling suppression.

Unlike the design presented in [8] where the cantilevers are located at the four corners of the proof mass, we use a pair of additional cantilevers symmetrically in the middle on all sides of the proof mass. These cantilevers can greatly enhance the decoupling efficiency which was proved by finite-element method (FEM) simulation and experimental verification presented in Section III. In addition, the sense capacitors are deliberately configured in a way that zero coupling can be achieved using a differential readout technique; this is explained in more detail below.

The proof mass is electrostatically excited to vibrate at its natural resonant frequency along the $x$ axis. On each of the two opposing sides along the $x$ axis of the proof mass are three sets of electrodes. The middle set is used as feedback electrodes for a closed loop drive mode scheme as described in Section IV, whereas the sets next to it are used to drive the proof mass into resonance. When an external rotation around the $z$ axis is applied as an input signal, a Coriolis force induced vibration is
TABLE I

DESIGN PARAMETERS OF THE GYROSCOPE

\begin{tabular}{|c|c|c|}
\hline Parameter & Drive mode & Sense mode \\
\hline width of cantilevers & $9 \mu \mathrm{m}$ & $9.2 \mathrm{um}$ \\
\hline spring constant of cantilevers & $1241 \mathrm{~N} / \mathrm{m}$ & $1304 \mathrm{~N} / \mathrm{m}$ \\
\hline comb finger length & $44 \mu \mathrm{m}$ & $20 \mu \mathrm{m}$ \\
\hline comb finger width & $4 \mu \mathrm{m}$ & $4 \mu \mathrm{m}$ \\
\hline nominal electrode overlap & $22 \mu \mathrm{m}$ & $10 \mu \mathrm{m}$ \\
\hline nominal capacitive gap & $5 \mu \mathrm{m}$ & $5 \mu \mathrm{m}$ \\
\hline number of fingers & 1600 & 2928 \\
\hline nominal capacitance & $9.97 \mathrm{pF}$ & $8.3 \mathrm{pF}$ \\
\hline number of feedback fingers & 224 & - \\
\hline nominal feedback capacitance & $1.4 \mathrm{pF}$ & - \\
\hline capacitive/deflection scale factor & $0.45 \mathrm{pF} / \mu \mathrm{m}$ & $0.83 \mathrm{pF} / \mu \mathrm{m}$ \\
\hline mass of proof mass & $1.979 \mu \mathrm{g}$ & $1.986 \mu \mathrm{g}$ \\
\hline
\end{tabular}

generated along the $y$ axis. The natural resonant frequency of the operating modes of the designed gyroscope can be calculated by

$$
f=\frac{1}{2 \pi} \sqrt{\frac{k}{m}}=\frac{1}{2 \pi} \sqrt{\frac{E}{\rho s} \cdot\left(\frac{b}{l}\right)^{3}}
$$

where $k$ is the overall spring constant of all the cantilevers, $m$ the mass of the movable part, $E$ Young's modulus of silicon, $s$ the relevant in-plane area, $\rho$ the density of silicon, $b$ the width, and $l$ the length of the suspension cantilevers, respectively. Usually, the structure thickness is predefined since it is related to the fabrication technology; in general, a large thickness is desirable for a heavy proof mass. The aspect ratio of the cantilevers, $b / l$, becomes the most critical parameter for the design of the operating modes.

The detailed geometric parameters of the gyroscope are listed in Table I.

In this design, the thickness of the proof mass and cantilevers is $80 \mu \mathrm{m}$ determined by the silicon-on-glass fabrication technology which is used as standard in the clean room laboratory at Peking University and is openly available. As an empirical compromise of the design parameters the analytical frequencies of drive and sense mode were chosen as $3987 \mathrm{~Hz}$ and $4080 \mathrm{~Hz}$ using (1), respectively. The difference is $93 \mathrm{~Hz}$ which results in the desired $|3 \mathrm{~dB}|$ bandwidth of about $50 \mathrm{~Hz}$ [11].

The sense electrodes are designed to be area-varying capacitors and thus move along the $y$ axis to measure the Coriolis induced motion. It is well known that the advantage of areavarying capacitors over gap-varying ones is lower air damping and better linearity, but at the cost of reduced sensitivity. Trading off these factors, area-varying capacitors are preferred in this design since nonvacuum packaging is used. The schematic view of Fig. 1 does not accurately depict the drive and sense electrodes configuration. Fig. 2 shows a more detailed view of the sense electrodes arrangement (which is similar to that of the drive 


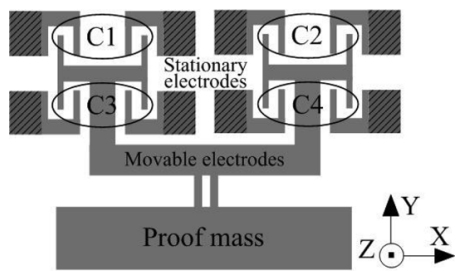

Fig. 2. Configuration of sense electrodes for differential readout scheme.

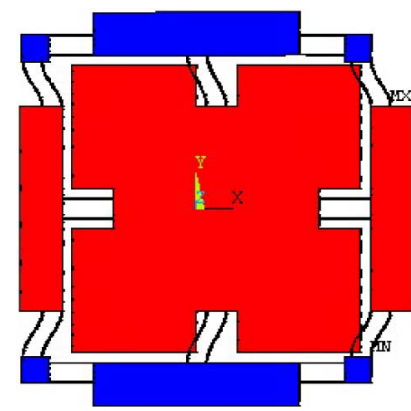

(a)

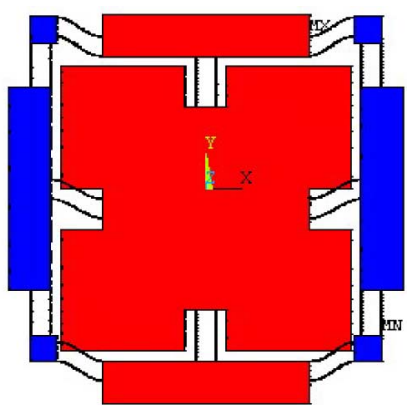

(b)

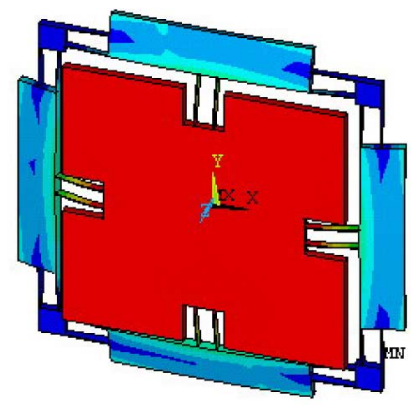

(c)

Fig. 3. Simulated modes of the gyroscope for a structural thickness of $h=80 \mu \mathrm{m}$ : (a) drive mode, (b) sense mode, and (c) out-of-plane third mode. (a) Drive mode: $f=3982 \mathrm{~Hz}$. (b) Sense mode: $f=4073 \mathrm{~Hz}$. (c) Third mode: $f=11236 \mathrm{~Hz}$.

electrodes). The sense capacitors are grouped into four symmetrical sets on either side of the proof mass; only the top half is shown in Fig. 2. Each set has a dedicated set of stationary electrodes, and form capacitors labeled $\mathrm{C} 1$ to $\mathrm{C} 4$ in Fig. 2. A motion in the positive sense direction $(+y)$ results in an increase of capacitors $\mathrm{C} 1$ and $\mathrm{C} 2$ whereas capacitors $\mathrm{C} 3$ and $\mathrm{C} 4$ decrease. The pick-off circuit detects $\Delta C=(C 1+C 2)-(C 3+C 4)$ providing a measure of the movement along the sense axis.

FEM simulations in ANSYS ${ }^{\mathrm{TM}}$ were used to verify the modal response of the designed structure. The two simulated fundamental modes were $3982 \mathrm{~Hz}$ and $4073 \mathrm{~Hz}$ which are in good agreement with the simple analytical model. To reduce complexity and increase computational efficiency the comb fingers for drive and sense mode were lumped together into an equivalent area. Fig. 3 illustrates the simulated modes of the gyroscope. It should be noted that both for the analytical calculations and ANSYS ${ }^{\mathrm{TM}}$ simulations fabrication imperfections were taken into account by reducing the cantilever width from the drawn value in the layout by approximately $1 \mu \mathrm{m}$; this value is based on measured data of previously fabricated devices. The third mode resonates out of plane with a frequency of $11,236 \mathrm{~Hz}$, which is much higher than those of the two operating modes;

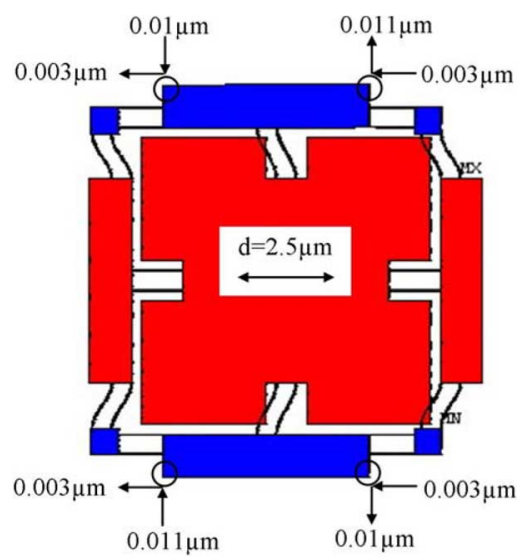

Fig. 4. Simulation of mechanical cross coupling between drive and sense mode.

therefore it does not degrade the performance. A simplifying assumption for (1) is that the thickness of the proof-mass $h$ is much larger compared to the width $(h \gg b)$. The value of parameter $h$ is critical for higher modes. In simulation, the resonant frequency of the third mode drops to $8610 \mathrm{~Hz}$ for a decreased thickness of $60 \mu \mathrm{m}$, and to $6407 \mathrm{~Hz}$ for a thickness of $40 \mu \mathrm{m}$. To minimize unwanted cross coupling between the two main operating modes and higher modes, it is preferred to have a structural thickness of more than $60 \mu \mathrm{m}$.

The mechanical cross coupling between drive and sense mode was simulated using ANSYS ${ }^{\mathrm{TM}}$ harmonic analysis. In the simulation, it was assumed the actual drive direction is aligned perfectly with the $x$ axis. The proof mass was driven to displace $2.5 \mu \mathrm{m}$ at the drive mode resonant frequency. This resulted in a displacement amplitude of the upper and lower sense electrodes in the four outer corners of $0.011 \mu \mathrm{m}$ and $0.003 \mu \mathrm{m}$ along the $x$ and $y$ axis, respectively. This simulation indicates that the mechanical cross coupling between drive and sense mode is less than $0.5 \%$ in the direction perpendicular to the drive direction, and about $0.1 \%$ along the drive direction, which are better than the result of 2\% demonstrated in [8]. As illustrated in Fig. 4, the motion in the sense direction ( $y$ axis) of the upper and lower sense electrodes is in anti-phase.

The motion of the movable electrodes of the sense mode due to mechanical cross-coupling results in a common mode electrical signal hence a zero output if a differential capacitive read-out circuit is used. This is the case for motion in $x$-direction as the movable electrodes on the top and bottom of the proof mass both move in the same direction with the same amplitude; therefore $C 1+C 2$ and $C 3+C 4$ change by the same amount. A similar argument applies for motion in the $y$-direction, where the left half of the movable electrodes on top of the proof mass moves up and the right half moves down, shown in Fig. 4-again resulting a zero differential capacitive signal.

The tiny discrepancy of displacement between the leftmost and rightmost corner is believed to be due to a numerical error in ANSYS $^{\mathrm{TM}}$. Due to the symmetric layout of the structure, the mechanical coupling from sense to drive mode is identical. Since the sense mode usually has a very small displacement, typically on the order of nanometer or even smaller, this effect is however negligible. 


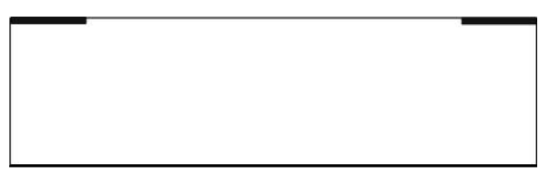

(a)

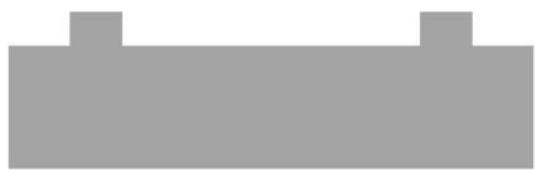

(b)

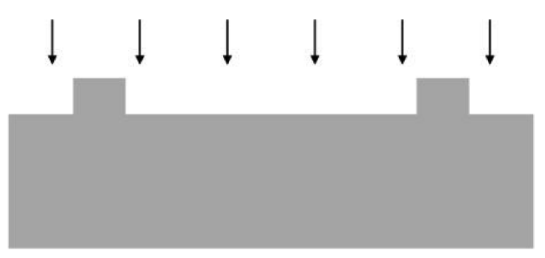

(c)

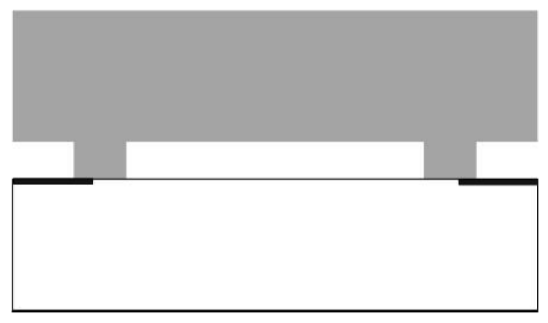

(d)

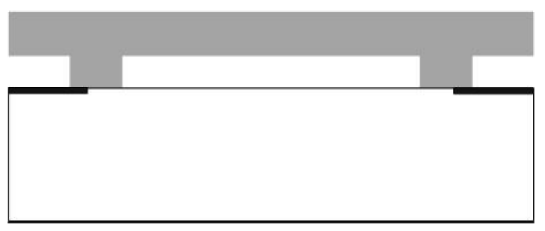

(e)

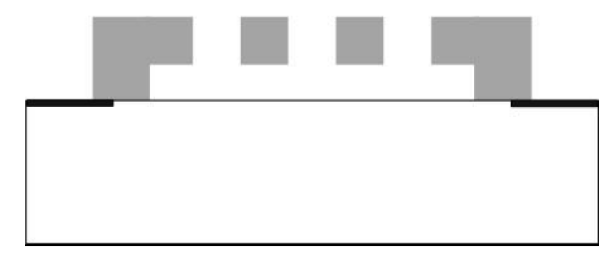

(f)

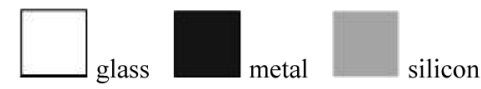

Fig. 5. Fabrication process; for detailed explanation see main text. (a) Interconnection forming. (b) Step etching. (c) Ion implantation. (d) Anodic bonding. (e) Silicon thinning. (f) Structure releasing.

\section{FABRICATION PROCESS}

A fabrication process based on silicon on glass (SOG) and deep reactive ion etching (DRIE) with an aspect ratio about 20 is utilized to fabricate the gyroscope to achieve a large and heavy proof mass and large capacitances. The starting wafers are a 4 inch highly doped silicon wafer with resistivity of $0.01-0.03 \Omega \cdot \mathrm{cm}$ and a Pyrex 7740 glass wafer with a thermal expansion coefficient approximately equal to silicon. It is a simple three-mask process with the schematic flow shown in Fig. 5. On the Pyrex wafer, a $200 \mathrm{~nm}$ thick Ti/Pt/Au layer is patterned by a liftoff process to make electrical interconnec-

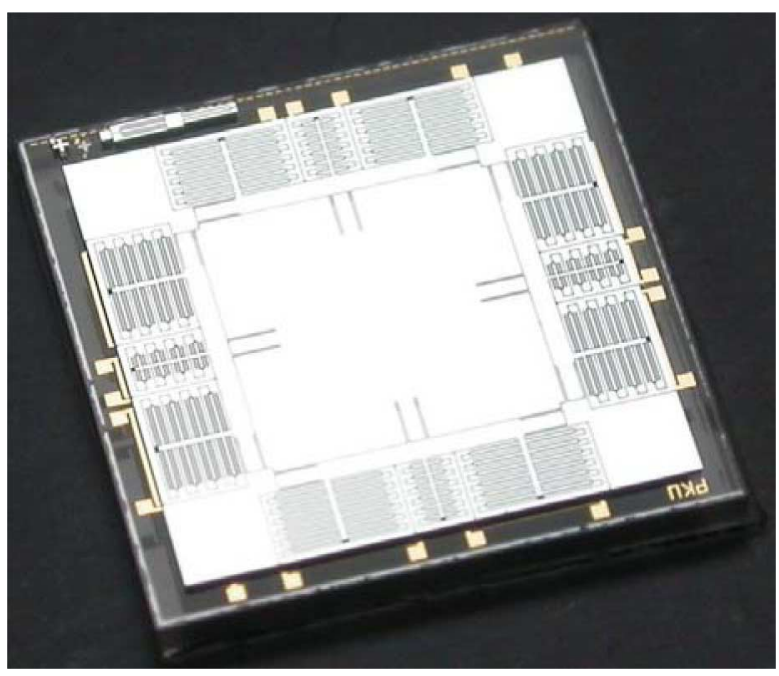

Fig. 6. Photograph of the gyroscope.

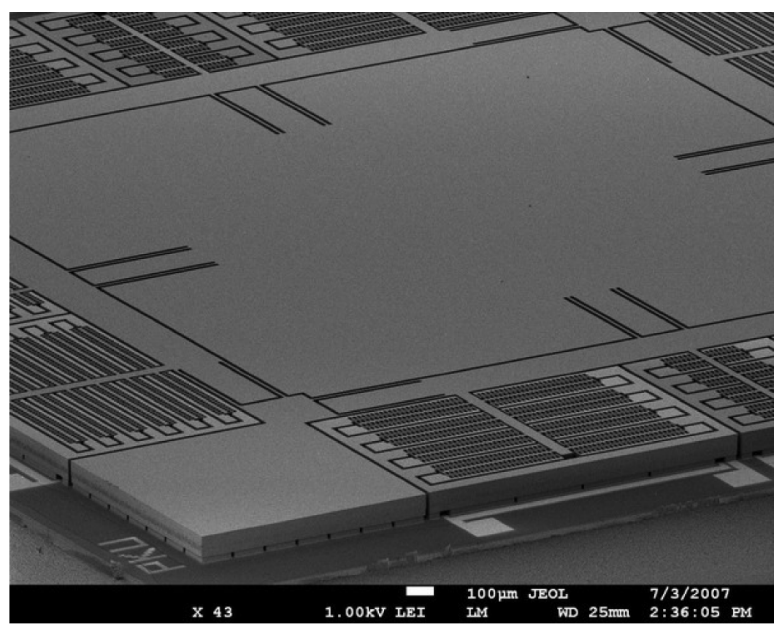

Fig. 7. SEM image of the gyroscope.

tions [Fig. 5(a)]. On the silicon wafer steps of $20 \mu \mathrm{m}$ height are etched by DRIE to define the anchor areas and the gap between the moving part and the substrate [Fig. 5(b)]. Then, the wafer is doped by phosphorus ion implantation to obtain good ohmic contacts at the anchor areas with the aforementioned electrical interconnections [Fig. 5(c)]. Next, the two wafers are anodically bonded together [Fig. 5(d)]. The silicon substrate is then thinned to about $100 \mu \mathrm{m}$ using potassium hydroxide $(\mathrm{KOH})$ [Fig. 5(e)]. Afterwards the gyroscope structures are released by a second DRIE step [Fig. 5(f)]. Finally, the wafers are diced and wire bonded. Figs. 6 and 7 show a photograph and scanning electron microscope (SEM) image of the fabricated gyroscope, respectively. The size of a chip is $6 \mathrm{~mm} \times 6 \mathrm{~mm}$ with an $80 \mu \mathrm{m}$-thick silicon structural layer.

\section{CONTROL AND INTERFACE CIRCUITRY}

In our previous work, an open loop drive scheme was adopted to be implemented with our gyroscope [9]. However, it cannot maintain the vibratory amplitude precisely, since the resonant frequency of the gyroscope drifts with environmental temperature variations, or other noise sources. For the gyroscope described in this paper, a self-oscillation drive interface with AGC 


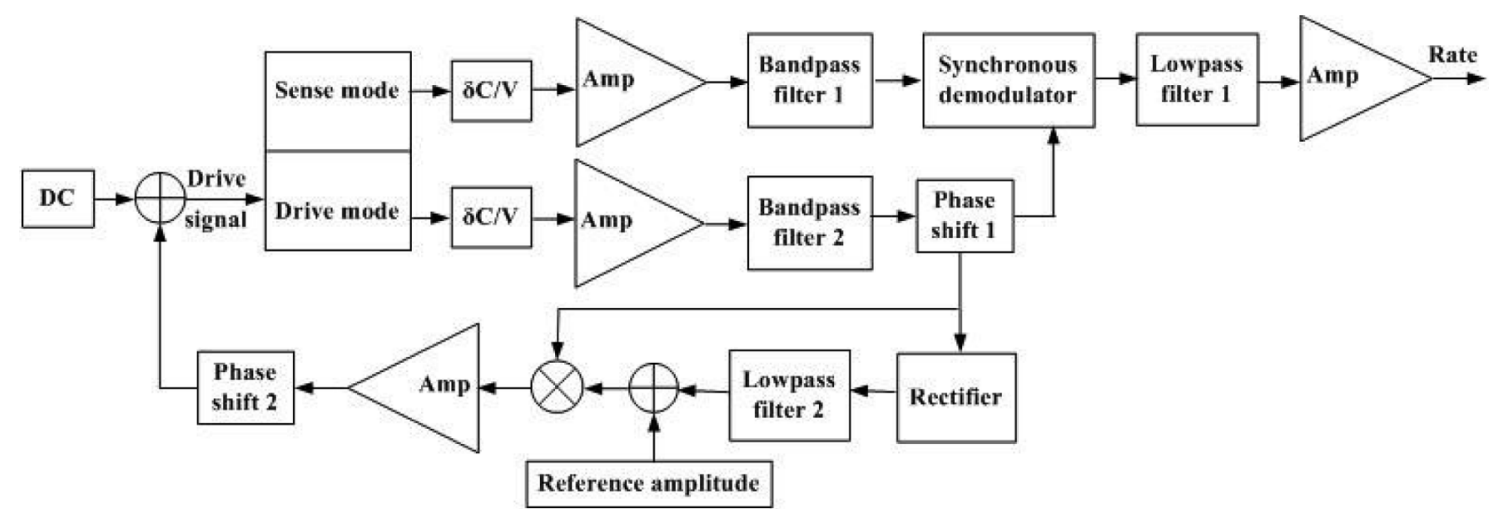

Fig. 8. Block diagram of the AGC drive mode control loop and the readout circuit for the operation of the gyroscope.

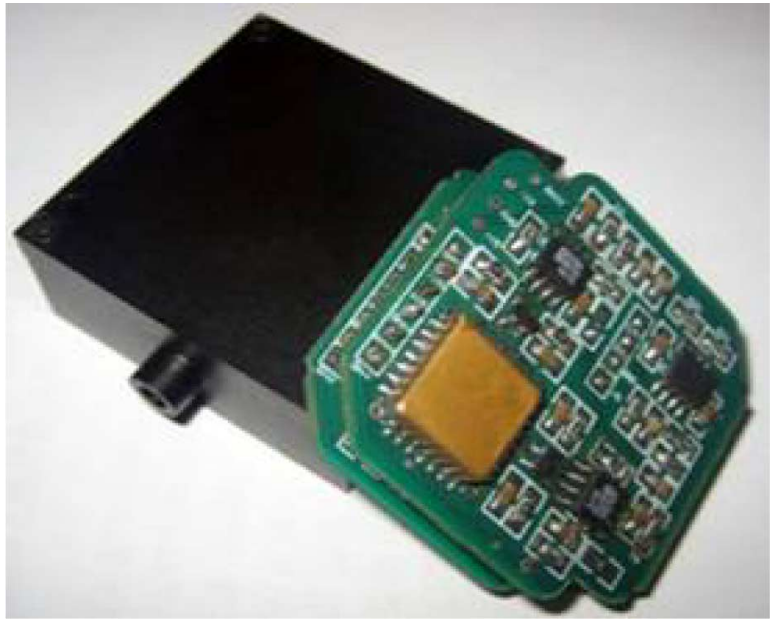

Fig. 9. Photograph showing the PCB implementation of the control and interface electronics for drive and sense mode.

(automatic gain controller) loop is designed to excite the gyroscope. The block diagram of both the drive and sense mode circuit is shown in Fig. 8, and the fabricated printed circuit board (PCB) in Fig. 9.

The drive mode self-oscillation system uses a rectifier and a low pass filter to obtain the peak value of the drive vibration amplitude and then compares it with a reference amplitude value. Phase shifter 1 is a first-order circuit based on a standard operational amplifier with a variable resistor allowing to adjust the phase angle between $0^{\circ}$ to $180^{\circ}$; it is used to compensate the phase delay of the band pass filter. Phase shifter 2 is optional, and can be used to compensate phase delay in the AGC; it can also be configured as a high-pass filter to restrict the noise bandwidth. When the system is in steady state, the error between the reference value and the detected amplitude is minimized. By dynamically adjusting the drive control loop damping, a self-oscillation with constant amplitude is sustained. If the measured amplitude is smaller than the desired reference amplitude, the AGC function will increase the loop gain to reduce the loop damping which causes the vibration amplitude to grow; conversely, if the vibration amplitude is larger than the reference amplitude, the AGC system will increase damping to reduce the vibration amplitude. The process keeps adjusting itself until the damping is equal to zero and the system will maintain a constant

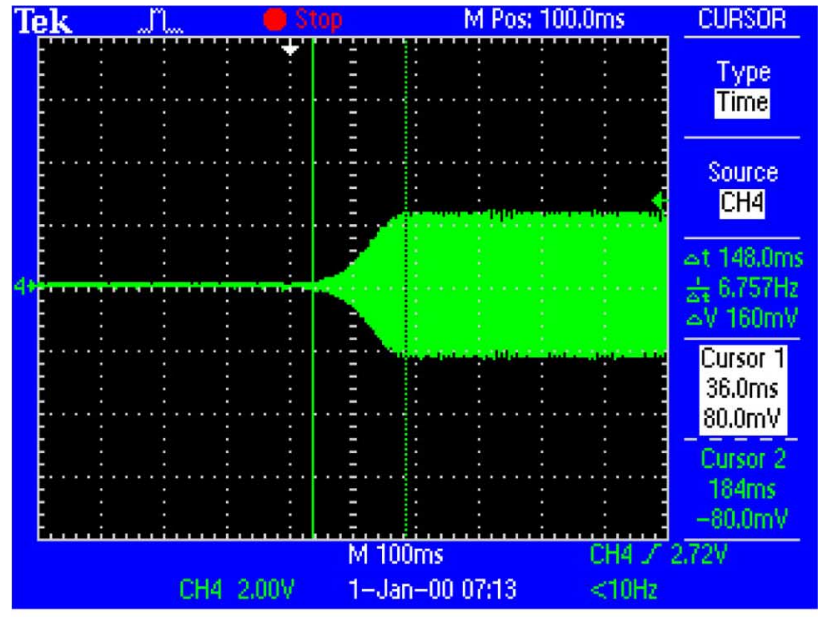

(a)

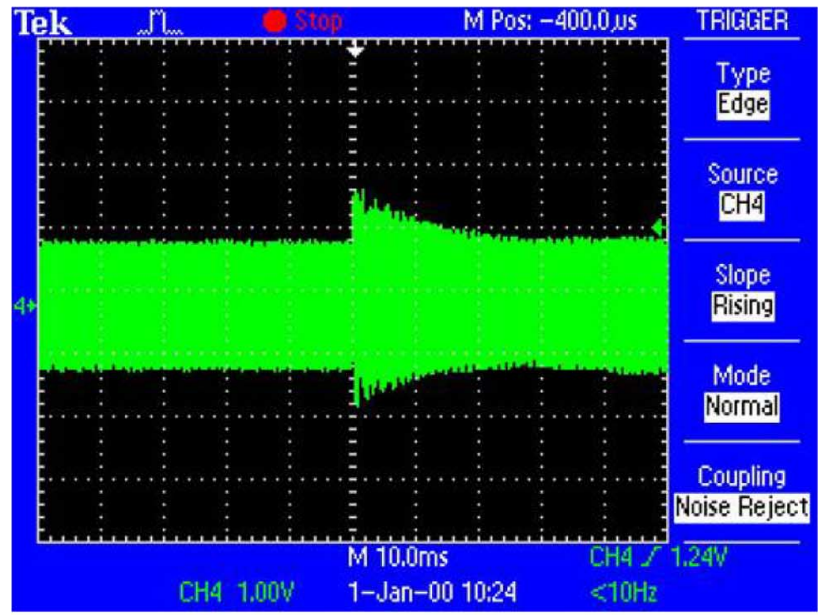

(b)

Fig. 10. Measured waveforms of the drive mode circuit: (a) self-oscillation waveform: reaching steady state after $150 \mathrm{~ms}$ from startup and (b) acceleration shock applied: the set value of the vibration amplitude is restored after $30 \mathrm{~ms}$.

amplitude oscillation. The steady-state vibration amplitude of the gyroscope drive mode is reached within $150 \mathrm{~ms}$ from the startup, as shown in Fig. 10(a). When an acceleration shock is applied to the PCB, the set value of the vibration amplitude is restored. Such a shock test is depicted in Fig. 10(b); after about $30 \mathrm{~ms}$ the vibration amplitude is restored. The shock magnitude 
was not measured in this experiment, thus this is only a qualitative test. Quantitative experiments were not carried out due to the lack of specialized shock testing equipment. The shock response strongly depends on the device package, and thus recovery to normal operation from a shock condition is difficult to predict theoretically. To reduce linear acceleration and shock susceptibility, it is advantageous to use two proof masses and drive them in anti-phase [12], [13]. Although our gyroscope is a single proof mass device, it would be relatively easy to put two devices on the same chip and have them operated in anti-phase. For shock survivability a simple argument can be put forward: The mechanical deflection per acceleration is about $0.03 \mu \mathrm{m} / \mathrm{g}$ for drive and sense mode, with a nominal electrode gap of $5 \mathrm{um}$, the maximum shock the gyroscope is therefore expected to withstand is about $170 \mathrm{~g}$.

The sense mode is a simple open loop system with a capacitive pick-off circuitry and phase-sensitive amplitude demodulator. A diode-ring is interfaced to the sense mode capacitors of the gyroscope, which is used to realize capacitive readout and demodulate the high-frequency electrical carrier applied to the proof mass. The circuit is described in more detail in [14]. The band pass filter 1 has a pass band of $\pm 50 \mathrm{~Hz}$ centered at the drive mode frequency; this removes frequency components due to the electrical excitation signal and low-frequency noise. Then, the signal is further demodulated by a synchronous demodulator, effectively multiplying the signal with the drive mode resonance frequency; this down-converts the angular rate input signal frequency to the base band. Any high-frequency components are finally removed by a second-order low pass filter with a $50 \mathrm{~Hz}$ cutoff frequency.

\section{IMPLEMENTATION AND EXPERIMENTAL RESULTS}

As an initial experiment, the mechanical resonant modes were determined. For the drive mode, the amplitude and phase response was measured by sweeping an AC drive signal applied to the drive electrodes and using the feedback electrodes for signal pick-off. In a similar manner, the amplitude and phase response of the sense mode was measured using the sense electrodes. Both Bode plots are shown in Fig. 11. The measured resonant frequency for the drive and sense mode is 3.359 and $3.444 \mathrm{kHz}$, respectively. In Fig. 11, two phenomena should be noted that the phases drop before the resonance and also they are not $90^{\circ}$ at the resonance. The reason is that a $\mathrm{RC}$ network is used in the diodering based capacitive readout interface [14]; therefore result in additional phase shift. Although fabrication imperfections were considered in the FEM simulation, they nevertheless reveal a considerable disagreement of more than $600 \mathrm{~Hz}$ between measured and simulated resonant frequency values. However, the frequency difference between drive and sense mode deviates only by $6 \mathrm{~Hz}$ from the simulated results; this indicates a systematic offset error across the wafer. It is well known that the bandwidth of the gyroscope output is approximately proportional to the frequency difference of drive and sense mode [11]; therefore the presented structural design is well suited to reduce the sensitivity of the gyroscope bandwidth to fabrication imperfections. Furthermore, the resonant frequencies of drive and sense modes have an approximately equal sensitivity to environmental

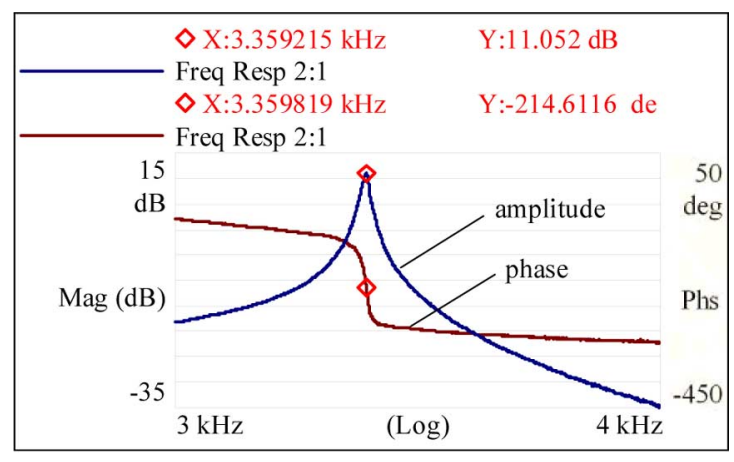

(a)

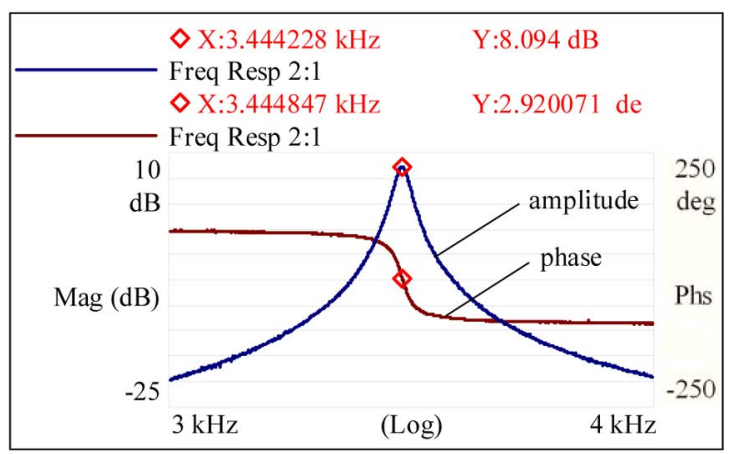

(b)

Fig. 11. Bode diagram of drive and sense mode, showing the amplitude and phase response: (a) drive mode and (b) sense mode.

parameters such as temperature due to the symmetric structure [8], therefore the frequency difference between drive and sense modes should remain constant. Consequently, the phase sensitivity is small as the phase angle is determined by the ratio of the drive and sense mode resonant frequencies.

The measured quality factors of the drive and sense mode at atmospheric pressure are 217 and 97, respectively. Based on the experimental results of resonant frequencies and quality factors, the bandwidth of the gyroscope is evaluated to be $34 \mathrm{~Hz}$ [15]. The discrepancy of the two quality factors is attributed to the difference in distance between the end of the movable electrodes and the anchor areas of the fixed electrodes. For a design with a frequency mismatch between sense and drive mode, the drive mode quality factor mainly determines the sensor performance. Therefore, the lower quality factor of the sense mode is not very detrimental.

Due to the difference between drive and sense mode resonant frequencies, the sense mode is not excited at its resonant frequency due to a Coriolis force. Therefore, the mechanical magnification factor for the displacement amplitude is not simply equal to the quality factor but is given by [11]

$$
\beta=\frac{1}{\sqrt{\left(1-\frac{\omega^{2}}{\omega_{n}^{2}}\right)^{2}+\left(\frac{\omega}{\omega_{n} \cdot Q}\right)^{2}}}
$$

where $\beta$ is the amplitude magnification factor; $\omega, \omega_{n}, Q$ are the operating frequency, natural resonant frequency and quality factor of the sense mode, respectively. If measured values are substituted into (2), the magnification factor for the sense mode 


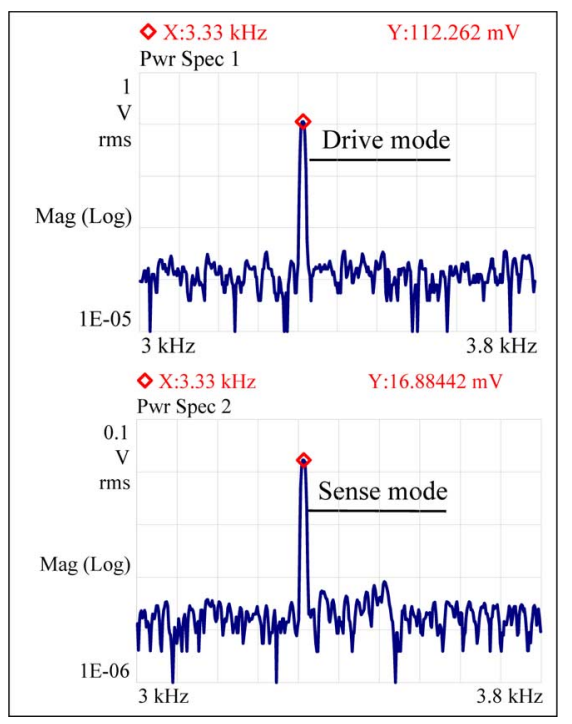

Fig. 12. Amplitudes of drive and sense mode output voltages for zero input rotational rate. The voltage amplitudes can be used to infer the mechanical cross coupling between drive and sense mode.

deflection amplitude is 20 . This is regarded as a good compromise between mechanical $Q$-factor amplification and bandwidth. Due to the relatively high $Q$-factors and amplitude magnification factor the gyroscope can achieve comparatively good performance for a MEMS device without vacuum packaging, which greatly reduces the fabrication cost. For the prototype device, the gyroscope was not hermetically packaged but simply glued in a standard PLCC (plastic leaded chip carrier) and protected by a cover.

The gyroscope was then tested using the aforementioned circuitry under atmospheric pressure. It was excited into resonance along the $x$-direction with a $1 \mathrm{Vp}$-p AC sinusoidal signal and a $10 \mathrm{~V}$ DC bias voltage. First, the mechanical cross coupling was evaluated by measuring the output of drive and sense mode simultaneously with no rotation input, as shown in Fig. 12. From the output voltage amplitudes it can be inferred that the sense mode displacement was equivalent to $1.35 \%$ of the drive mode displacement (for the conversion from the measured output voltage amplitude to displacement, see Appendix A). The drive to sense mode cross coupling was approximately 2.7 times higher than the simulated values which was less than $0.5 \%$; this can be explained by quadrature error and asymmetric defects caused by fabrication imperfections which were not considered in the simulation. In the simulation, it was assumed that the drive direction is perfectly aligned to the $x$ axis which is not the case in the fabricated device leading to quadrature error. Additionally, asymmetric defects lead to an increase of mechanical cross coupling [16], therefore common mode errors do not ideally cancel as intended by the design; this further increases the mechanical cross coupling.

Second, the $z$ axis gyroscope was tested on a rate table. Fig. 13 shows the output characteristic of the gyroscope for DC angular rate signals. In the range of $\pm 300^{\circ} / \mathrm{s}$, the achieved scale factor was $10.7 \mathrm{mV} / \%$ s with a $R^{2}$-nonlinearity of $0.12 \%$.

Fig. 14 shows the output signal of the gyroscope at an angular vibration input with an amplitude of $12.5^{\circ} / \mathrm{s}$ and a frequency of

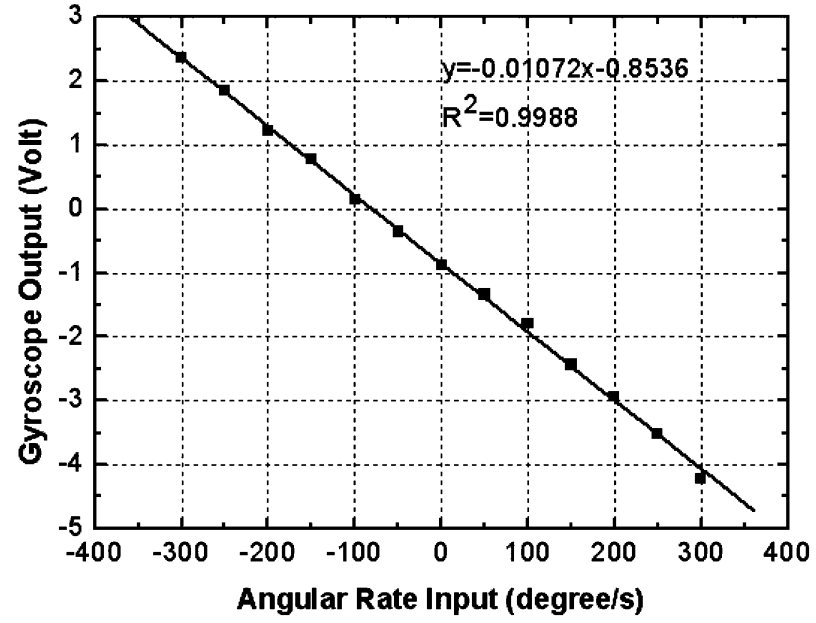

Fig. 13. Measured output signals of the gyroscope for DC angular rate inputs in a range of $\pm 300^{\circ} / \mathrm{s}$.

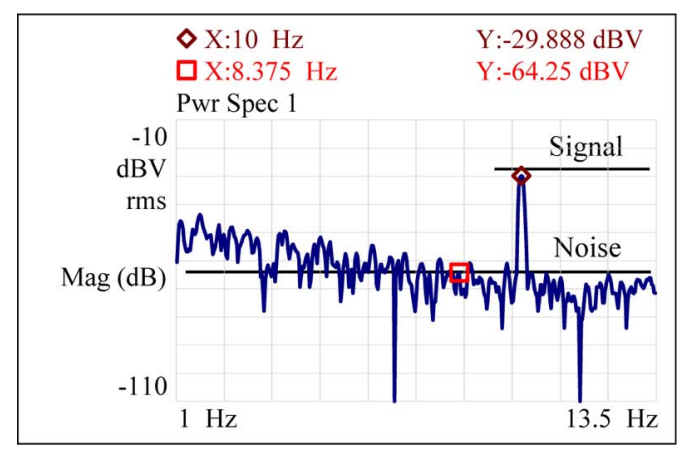

Fig. 14. Output voltage spectrum when the gyroscope was mounted on a rate table producing an angular rate signal with an amplitude of $12.5^{\circ} / \mathrm{s}$ and a frequency of $10 \mathrm{~Hz}$.

$10 \mathrm{~Hz}$. The noise floor is at approximately $-64 \mathrm{~dB}$ resulting in a signal-to-noise ratio (SNR) of about $35 \mathrm{~dB}$. The $\mathrm{SNR}$ can be further improved by properly shielding the electromagnetic interference from the rate table as it was obvious from signals probed on an oscilloscope that operation of the rate table significantly degraded signal quality.

Fig. 15 shows the spectrum of the sense mode signal after the band pass filter 1 (referring to Fig. 8) in response to an angular rate input with an amplitude of $12.5^{\circ} / \mathrm{s}$ and a frequency of $10 \mathrm{~Hz}$. Clearly, a quadrature signal is visible with a magnitude equivalence of $265^{\circ} / \mathrm{s}$. This is filtered out by the synchronous demodulation.

Fig. 16 shows the noise spectrum of the gyroscope for zero angular rate input signal. The root noise power spectral density equivalent angular rate is $0.0015^{\circ} / \mathrm{s} / \mathrm{Hz}^{1 / 2}\left(=5.4^{\circ} / \mathrm{h} / \mathrm{Hz}^{1 / 2}\right)$ in a $50 \mathrm{~Hz}$ bandwidth.

Without any active temperature control, the $1-\sigma$ bias stability is $4^{\circ} / \mathrm{s}$ for a long-term measurement over eight hours; if the sensor is left to settle down for two hours the bias stability improves to $1^{\circ} / \mathrm{s}$ for the remaining 6 hours measurement. For a 120 second short-time measurement the bias stability is $0.3^{\circ} / \mathrm{s}$. These numbers were derived from Fig. 17 showing an 8 hours measurement. 


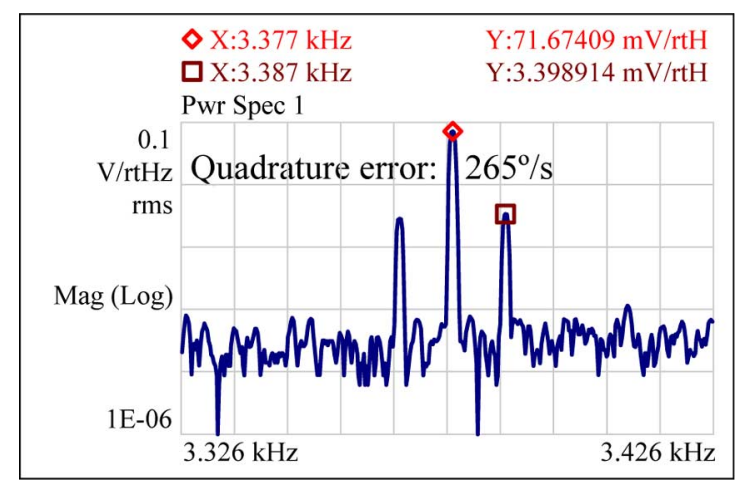

Fig. 15. Measured output of the sense mode before synchronous demodulation in response to an angular rate input with an amplitude of $12.5^{\circ} / \mathrm{s}$ and a frequency of $10 \mathrm{~Hz}$, showing a quadrature signal equivalent to $265^{\circ} \%$ s.

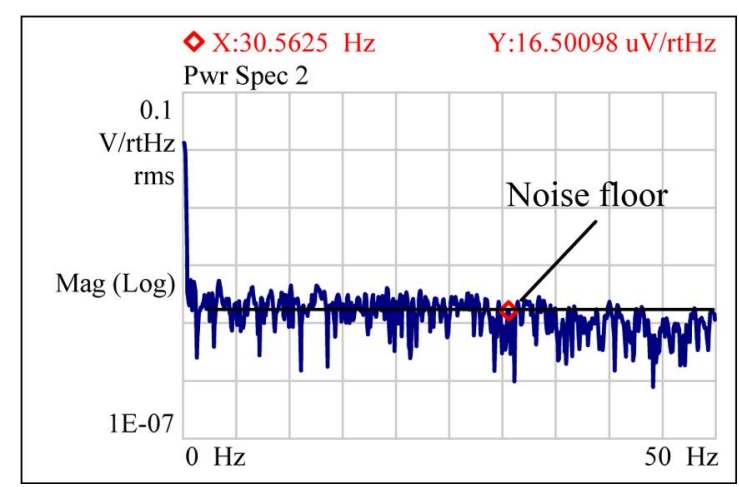

Fig. 16. Noise performance of the gyroscope, showing the output signal spectrum of the pick-off circuit without an angular rate input signal. The RMS noise floor is about $16.5 \mathrm{uV} / \mathrm{Hz}^{1 / 2}$ in a $50 \mathrm{~Hz}$ bandwidth.

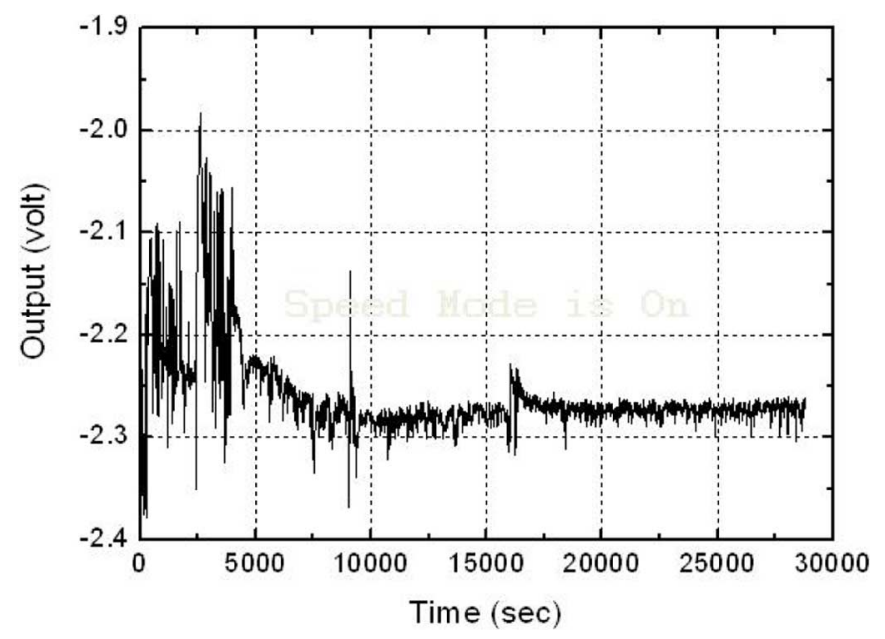

Fig. 17. Output signal—bias stability over a measurement of 8 hours with zero rotational input signal applied to the gyroscope.

\section{CONCLUSION}

This paper presents a high-resolution silicon-on-glass $z$ axis gyroscope operating at atmospheric pressure. Using two groups of $1 \mathrm{DOF}$ cantilevers to suspend the 2DOF proof mass, the designed structure realizes a double-decoupling scheme which efficiently suppresses cross coupling between drive and sense mode. A highly symmetric structural design is chosen to decrease the sensitivity of the gyroscope bandwidth to fabrication imperfections. FEM simulations predict the cross coupling to be as low as $0.5 \%$ and experimental measurement data indicate a value of $1.35 \%$. The higher value is attributed to quadrature error and asymmetries due to fabrication tolerances. Experimental results show that although the measured resonant frequencies of the drive and sense mode deviates more than $600 \mathrm{~Hz}$ from the intended design values, the frequency difference disagrees only by $6 \mathrm{~Hz}$. The combination of a large proof mass and the usage of low air damping electrodes achieve a large quality factor of 217 for the drive mode and 97 for the sense mode, making the operation of the gyroscope suitable at atmospheric pressure which greatly reduces the overall cost of the sensor. The measured scale factor of the gyroscope is $10.7 \mathrm{mv} /{ }^{\circ} / \mathrm{s}$ in a range of $\pm 300^{\circ} / \mathrm{s}$ with a $R^{2}$-nonlearity of $0.12 \%$. The noise performance of the gyroscope was determined as a root power spectral density noise equivalent angular rate of $0.0015^{\circ} / \mathrm{s} / \mathrm{Hz}^{1 / 2}\left(=5.4^{\circ} / \mathrm{h} / \mathrm{Hz}^{1 / 2}\right)$ in a $50 \mathrm{~Hz}$ bandwidth; this makes it suitable to meet the requirements of medium-precision applications with the potential to replace vacuum packaged gyroscopes. Without any active temperature control, the $1-\sigma$ bias stability can achieve $1^{\circ} / \mathrm{s}$ for a 6-hour measurement and $0.3^{\circ} / \mathrm{s}$ for a 120 -second short-time measurement.

As a next step, the gyroscope will be put in a hermetically sealed package, which is expected to reduce considerably bias scale factor and long-term drift.

\section{APPENDIX}

TRANSFORM FROM VOLTAGE TO DisPlaCEMENT

The original voltage outputs of drive and sense mode before they are amplified by the operational amplifiers are given by

$$
\begin{aligned}
& \frac{1}{\frac{49400}{R_{G 1}+1}} \times 112.262=1.147 \mathrm{mV} \\
& \frac{1}{\frac{49400}{R_{G 2}+1}} \times 16.8844=0.034 \mathrm{mV} \\
& \text {. }
\end{aligned}
$$

respectively, where $R_{G 1}=510 \Omega, R_{G 2}=100 \Omega$ are external gain setting resistors of amplifier AD620 for drive and sense mode, respectively.

Because of $\delta C / C=\delta L / L$, consequently, the equation of displacement ratio relevant to voltages is

$$
\frac{\frac{\delta L_{s}}{L_{s}}}{\frac{\delta L_{d}}{L_{d}}}=\frac{\frac{\delta L_{s}}{10}}{\frac{\delta L_{d}}{22}}=\frac{0.034}{1.147} .
$$

Thus, the resultant mechanical coupling in displacement is

$$
\frac{\delta L_{s}}{\delta L_{d}}=1.35 \%
$$

where $C, \delta C$ are the nominal capacitance of the sense mode and capacitance change; $\delta L_{s}, \delta L_{d}$ are the displacements of sense and drive electrodes; $L_{s}, L_{d}$ are the overlap of sense and drive electrodes, respectively.

\section{REFERENCES}

[1] S. H. Kim, Y. K. Kim, J. W. Song, and J. G. Lee, "A surface-bulkmicromachined electromagnetic gyroscope operating at atmospheric pressure," Jpn. J. Appl. Phys., vol. 39, pp. 7130-7133, 2000. 
[2] S. H. Kim, J. Y. Lee, C. H. Kim, and Y. K. Kim, "A bulk-micromachined single crystal silicon gyroscope operating at atmospheric pressure," in Proc. Transducers'01, Munich, Germany, 2001, pp. 476-479.

[3] H. K. Xie and G. K. Fedder, "A DRIE CMOS-MEMS gyroscope," in Proc. IEEE Sensors, Orlando, FL, 2002, pp. 1413-1418.

[4] B. Xiong, L. Che, and Y. Wang, "A novel bulk micromachined gyroscope with slots structure working at atmosphere," Sens. Actuators A 107, pp. 137-145, 2003.

[5] Y. Chen, J. Jiao, B. Xiong, L. Che, X. Li, and Y. Wang, "A novel tuning fork gyroscope with high $Q$-factors working at atmospheric pressure," Microsystem Technol., pp. 111-116, 2005.

[6] C. Acar and A. M. Shkel, "Inherently robust micromachined gyroscopes with 2-DOF sense-mode oscillator," J. Microelectromech. Syst., vol. 15 , pp. 380-387, 2006.

[7] S. E. Alper, K. Azgin, and T. Akin, "High-performance SOI-MEMS gyroscope with decoupled oscillation modes," in Proc. IEEE MEMS'06, Istanbul, Turkey, 2006, pp. 70-73.

[8] S. E. Alper and T. Akin, "A single-crystal silicon symmetrical and decoupled MEMS gyroscope on an insulating substrate," J. Microelectromech. Syst., vol. 14, pp. 707-717, 2005.

[9] H. T. Ding, X. S. Liu, J. Cui, X. Z. Chi, Z. C. Yang, and G. Z. Yan, “A bulk micromachined $z$-axis single crystal silicon gyroscope for commercial applications," in Proc. IEEE NEMS, Sanya, China, 2008, pp. 1039-1042.

[10] H. T. Ding, J. Cui, X. S. Liu, X. Z. Chi, Z. C. Yang, and G. Z. Yan, "A highly double-decoupled self-oscillation gyroscope operating at atmospheric pressure," in Proc. IEEE Sensor, Lecce, Italy, 2008, pp. 674-677.

[11] M. H. Bao, "Micro mechanical transducers: Pressure sensors," in Accelerometers and Gyroscopes. Amsterdam, The Netherlands: Elsevier, 2000, pp. 365-369.

[12] J. Bernstein, S. Cho, A. T. King, A. Kourepenis, P. Maciel, and M. Weinberg, "A micromachined comb-drive tuning fork rate gyroscope," in Proc. IEEE MEMS, Fort Lauderdale, FL, 1993, pp. 143-148.

[13] A. R. Schofield, A. A. Trusov, C. Acar, and A. M. Shkel, "Anti-phase driven rate gyroscope with multi-degree of freedom sense mode," in Proc. Transducers, Lyon, France, 2007, pp. 1199-1202.

[14] J. Wang, L. Qian, and G. Yan, "CMOS-MEMS gyroscope using integrated diode-rings as interface circuits," in Proc. IEEE ICSICT'08, Beijing, China, 2008, pp. 2432-2435.

[15] H. K. Xie, "Gyroscope and micromirror design using vertical-axis CMOS-MEMS actuation and sensing," Ph.D. dissertation, Carnegie Mellon Univ., Pittsburgh, PA, 2002.

[16] B. Lv, X. S. Liu, Z. C. Yang, and G. Z. Yan, "Simulation of a novel lateral axis micromachined gyroscope in the presence of fabrication imperfections," Microsystem Technol., pp. 711-718, 2008.

Haitao Ding was born in Shandong, China, in 1979. He is working towards the Ph.D. degree at the Department of Microelectronics, Peking University, Beijing, China.

His research interests include design, fabrication and characterization of microgyroscopes, and sigma-delta modulator-based control systems for MEMS inertial sensors.

Xuesong Liu was born in Hebei, China, in 1980. He received the B.S. and Ph.D. degrees in microelectronics from Peking University, Beijing, China, in 2003 and 2009 , respectively.

He is now working as a Faculty Member at the Institute of Mechanics, Chinese Academy of Sciences. His current interests include microgyroscopes and microaccelerometers.
Longtao Lin was born in Heilongjiang, China, in 1978. He received the B.S. and M.S. degrees in mechanical engineering from the Harbin Institute of Technology, Harbin, China, in 2001 and 2003, respectively.

Then, he worked at the Beijing Institute of Computer Application and Simulation Technology Sciences for three years. In 2006, he joined the Institute of Microelectronics at Peking University as a technical staff member. His research interest focuses on the design of readout circuits for MEMS sensors.

Xiaozhu Chi was born in Shandong, China, in 1968. She received the B.S. degree in electronics engineering from the Harbin Institute of Technology, Harbin, China, in 1990, and the Ph.D. degree from Harbin Engineering University, Harbin, in 2004

She worked as a Postdoc Staff at the Institute of Microelectronics, Peking University, from 2006 to 2009. She is now working as a Senior Engineer at the China Meteorological Administration.

Jian Cui was born in Shenyang, China, in 1982. He received the B.S. degree (First Class Honors) in electrical and mechanical engineering from Beijing Jiaotong University, Beijing, China. He is currently working towards the Ph.D. degree at the Institute of Microelectronics, Peking University, Beijing.

His main research interests are design of electronics and control strategies for MEMS sensors.

Michael Kraft received the Dipl.-Ing. (Univ.) degree in electrical and electronics engineering from the Friedrich Alexander Universitat, Erlangen-Nürnberg, Germany, in 1993, and the Ph.D. degree from Coventry University, Coventry, U.K., in 1997.

He spent two years at the Berkeley Sensors and Actuator Center, University of California, Berkeley, from 1997 to 1999, working on the design of integrated micromachined gyroscopes. He is currently a Professor with the School of Electronics and Computer Science, University of Southampton, Southampton, U.K. His principle interests include micromachined inertial sensors, MEMS sensors and actuators, intelligent control systems for MEMS devices, and electronic circuit design.

Zhenchuan Yang received the B.S. and Ph.D. degrees at the School of Electronics Engineering and Computer Science, Peking University, Beijing, China, in 1998 and 2004, respectively.

From 2004 to 2006, he was a Research Associate at the Department of Electronics and Computer Engineering, Hong Kong University of Science and Technology, engaging in researches on GaN-based MEMS devices. In 2006, he joined the Institute of Microelectronics, Peking University, where he is currently an Associate Professor. His research interests include micromachined inertial sensors, monolithic integration process and GaN-based MEMS devices.

Guizhen Yan received the B.S. degree in microelectronics from Peking University, Beijing, China, in 1974.

She is now a Professor at the Institute of Microelectronics, Peking University. Her research interests include design and fabrication of MEMS inertial sensors, monolithic integration of bulk MEMS sensors and IC. She has published more than 150 technical papers, and filed about 20 patents. 